\title{
Ru-Co-Mn trimetallic alloy nanocatalyst driving bifunctional redox electrocatalysis
}

\author{
Shan Liu, Erhuan Zhang*, Xiaodong Wan, Rongrong Pan, Yuemei Li, Xiuming Zhang, Mengyao Su, \\ Jia Liu* and Jiatao Zhang*
}

\begin{abstract}
Water electrolysis is one of the most promising approaches for producing hydrogen. However, it has been hindered by the sluggishness of the anodic oxygen evolution reaction. In this work, we fabricated $\mathrm{Ru}-\mathrm{Co}-\mathrm{Mn}$ trimetallic alloy nanoparticles on $\mathrm{N}$-doped carbon support (RuCoMn@ NC) via the pyrolysis-adsorption-pyrolysis process using ZIF67 as a precursor. The RuCoMn@NC catalyst exhibited excellent electrocatalytic performance for the hydrogen evolution reaction (HER) over a wide range of $\mathrm{pH}$ and glucose oxidation reaction in alkaline media. It showed exceptional HER activity in alkaline medium, superior to that of the commercial $\mathrm{Pt} / \mathrm{C}$ catalyst $(20 \mathrm{wt} \%)$, and good electrochemical stability. Further, a two-electrode alkaline electrolyzer pairing RuCoMn@NC as both cathode and anode was employed, and only a cell voltage of $1.63 \mathrm{~V}$ was required to attain a current density of $10 \mathrm{~mA} \mathrm{~cm}^{-2}$ in glucose electrolysis, which is about $270 \mathrm{mV}$ lower than that in the overall water-splitting electrolyzer. This paper provides a promising method for developing efficiently bifunctional electrocatalysts driving redox electrocatalysis, and it would be beneficial to energy-saving electrolytic $\mathrm{H}_{2}$ production.
\end{abstract}

Keywords: Ru-Co-Mn trimetallic alloy, HER, glucose oxidation reaction, redox electrocatalysis

\section{INTRODUCTION}

The energy crisis is one of the critical issues limiting human development, and the key to solving the problem is the design and synthesis of high-performance catalytic materials for clean energy generation and conversion [1]. Hydrogen is considered a clean energy source that can replace fossil fuels in the future. It has high combustion calorific value and high weight energy density [2]. Its combustion product is water, and the energy conversion process is clean and pollution-free. Significantly, hydrogen can be made from water through electrolytic water splitting, which is widely considered as an environmentally friendly and sustainable technology for producing clean energy. Water electrolysis involves two semi-reactions: hydrogen evolution reaction (HER) and oxygen evolution reaction (OER). However, as a key step for water decomposition, OER has slow reaction kinetics and high overpotential, which hinder the progress of the overall water splitting. The major energy loss in water electrolysis is attributed to the sluggishness of anodic OER [3]. Recently, some researchers have proposed hydrogen production via oxidation reactions of substances, such as hydrazine [4], urea [5], and 5-hydroxymethyl furfural [6], instead of anodic OER. The voltage input of the electrolytic cell can be reduced by anodic organic molecular oxidation, which is more favorable in thermodynamics than OER [6-9]. The theoretical thermodynamic potential of glucose oxidation to gluconate is much smaller than that of OER $(1.23 \mathrm{~V})$ [10], making it an attractive substitute for OER in hydrolysis [11,12]. It is vital to design electrode materials with high catalytic activity and stability that can drive redox reactions to greatly reduce the overpotential of cathode and anode, thereby decreasing the energy consumption of industrial electrocatalytic production of hydrogen $[13,14]$.

Currently, platinum (Pt) is the most commonly used commercial HER electrocatalyst owing to its excellent catalytic performance. However, its large-scale commercialization is limited by its high cost, low abundance, and poor durability [15]. In the past few decades, several transition-metal (TM)-based catalysts, including their carbides [16], nitrides [17], sulfides [18], phosphides [19,20], oxides [21], borides [22], and heterogeneous heteroatomic (, P, S, B) [23-26] doped carbon materials have demonstrated good capacity for driving HER. They are promising alternative catalysts for HER owing to their low cost, high efficiency, and abundance. Nevertheless, the catalytic performance of TM-based catalysts is still unsatisfactory compared with that of Pt-based electrocatalysts. Recently, metal alloy catalysts containing small amounts of noble metals have attracted attention owing to their good corrosion resistance in acidic and alkaline environments [27]. Alloying of transition metals with relatively cheap non-Pt noble metals can combine their distinct advantages, and thus, the synthesized catalyst exhibits a high performance-to-price ratio. Besides, metalorganic frameworks (MOFs) and other coordination compounds have recently gained wide attention as precursors for preparing carbon-based electrocatalysts with high electrical conductivity and uniform distribution of active sites [28-30]. The resulting $\mathrm{N}$-doped carbon matrix can afford a good platform to disperse alloying metal particles.

Herein, we developed a Ru-Co-Mn ternary alloy supported on $\mathrm{N}$-doped porous carbon polyhedral (RuCoMn@NC), which can

School of Materials Science \& Engineering, Beijing Key Laboratory of Construction-Tailorable Advanced Functional Materials and Green Applications, Experimental Center of Advanced Materials, Beijing Institute of Technology, Beijing 100081, China

*Corresponding authors (emails: zhangjt@bit.edu.cn (Zhang J); liujia86@bit.edu.cn (Liu J); erhuanzh@163.com (Zhang E)) 
function as a highly efficient bifunctional electrocatalyst for both HER and glucose oxidation reaction (GOR). Owing to the alloying effect with favorable composition, abundant mesoporous structure, large surface area, and conductive N-doped carbon matrices, the optimized RuCoMn@NC showed excellent HER performance under all $\mathrm{pH}$ conditions and good GOR performance in alkaline media. The RuCoMn@NC catalyst displayed an overpotential of $38 \mathrm{mV}$ at $10 \mathrm{~mA} \mathrm{~cm}^{-2}$ in $1.0 \mathrm{~mol} \mathrm{~L}^{-1}$ $\mathrm{KOH}$, exceeding that of the commercial $20 \mathrm{wt} \% \mathrm{Pt} / \mathrm{C}$ catalyst $(64 \mathrm{mV})$. Moreover, the overpotentials were 119 and $90 \mathrm{mV}$ to deliver a current density of $10 \mathrm{~mA} \mathrm{~cm}^{-2}$ in $0.5 \mathrm{~mol} \mathrm{~L}^{-1} \mathrm{H}_{2} \mathrm{SO}_{4}$ and $1.0 \mathrm{~mol} \mathrm{~L}^{-1}$ phosphate buffer saline (PBS). An electrolyzer with RuCoMn@NC electrode as both HER cathode and GOR anode required only an applied voltage of $1.63 \mathrm{~V}$ for an electrolysis current density of $10 \mathrm{~mA} \mathrm{~cm}^{-2}$, which was $270 \mathrm{mV}$ less than that for water splitting.

\section{EXPERIMENTAL SECTION}

\section{Synthesis of ZIF-67}

The ZIF-67 particles were synthesized following the procedure reported in a previous study [31] with some modifications. The process was as follows: $\mathrm{Co}\left(\mathrm{NO}_{3}\right)_{2} \cdot 6 \mathrm{H}_{2} \mathrm{O}(1.726 \mathrm{~g}, 6 \mathrm{mmol})$ and 2methylimidazole (2-MeIm, $1.970 \mathrm{~g}, 24 \mathrm{mmol}$ ) were, respectively, dissolved in $30 \mathrm{~mL}$ of methanol to form clear solutions; the prepared 2-MeIm solution was quickly poured into a $\mathrm{Co}^{2+}$ containing solution and stirred for $10 \mathrm{~s}$; the mixed solution was maintained at room temperature for $20 \mathrm{~h}$; the resulting ZIF-67 precipitates were collected by centrifugation, washed with ethanol for several times, and dried at $60^{\circ} \mathrm{C}$ in the vacuum oven for $24 \mathrm{~h}$.

\section{Synthesis of the Co@NC}

The as-synthesized ZIF-67 was poured into a tube furnace and heated to $400^{\circ} \mathrm{C}$ at a ramp rate of $2^{\circ} \mathrm{C} \min ^{-1}$ and kept for $2 \mathrm{~h}$ in a 5 vol\% $\mathrm{H}_{2} / \mathrm{Ar}$ flow. The temperature was then raised to $700^{\circ} \mathrm{C}$ with a ramp rate of $2^{\circ} \mathrm{C} \mathrm{min}{ }^{-1}$ and maintained at the temperature for another $2 \mathrm{~h}$. The furnace was then cooled naturally to room temperature $\left(25^{\circ} \mathrm{C}\right)$ to obtain Co@NC black powder.

\section{Synthesis of RuCoMn@NC}

For RuCoMn@NC, $75 \mathrm{mg}$ of the as-prepared Co@NC nanoparticles (NPs) were dispersed in $5 \mathrm{~mL}$ of $n$-hexane and ultrasonicated at room temperature for $10 \mathrm{~min}$ to get a homogeneous dispersion. Then, $200 \mu \mathrm{L}$ of a $\mathrm{RuCl}_{3} \cdot 3 \mathrm{H}_{2} \mathrm{O}$ aqueous solution $\left(1.55 \mathrm{~mol} \mathrm{~L}^{-1}\right)$ and $200 \mu \mathrm{L}$ of a $\mathrm{MnCl}_{2} \cdot 4 \mathrm{H}_{2} \mathrm{O}$ aqueous solution $\left(1.55 \mathrm{~mol} \mathrm{~L}^{-1}\right)$ were added to the above solution dropwise and stirred for $2 \mathrm{~h}$. Co@NC with the adsorbed $\mathrm{Ru}^{3+}$ and $\mathrm{Mn}^{2+}$ was collected by centrifugation and then freeze-dried for $24 \mathrm{~h}$. The dried sample was placed in a tube furnace and heated to $500^{\circ} \mathrm{C}$ at a ramp rate of $2^{\circ} \mathrm{C} \mathrm{min}^{-1}$ and kept for $2 \mathrm{~h}$ in a $5 \mathrm{vol} \% \mathrm{H}_{2} / \mathrm{Ar}$ flow. The same synthetic procedure was employed to synthesize $\mathrm{RuCo@NC}$ without using the $\mathrm{MnCl}_{2} \cdot 4 \mathrm{H}_{2} \mathrm{O}$ aqueous solution. For RuCoFe@NC, RuCoNi@NC, and RuCoCu@NC, the $\mathrm{MnCl}_{2} \cdot 4 \mathrm{H}_{2} \mathrm{O}$ aqueous solution was replaced with $\mathrm{Fe}\left(\mathrm{NO}_{3}\right)_{3}$. $9 \mathrm{H}_{2} \mathrm{O}, \mathrm{Ni}\left(\mathrm{NO}_{3}\right)_{2} \cdot 6 \mathrm{H}_{2} \mathrm{O}$, and $\mathrm{CuCl}_{2} \cdot 2 \mathrm{H}_{2} \mathrm{O}$ aqueous solutions, respectively.

\section{Catalyst characterization}

Low-resolution transmission electron microscopy (TEM) images of the samples were obtained using HITACHI H-7650 (accelerating voltage of $80 \mathrm{kV}$ ) electron microscope. High-resolution TEM (HR-TEM) and element mapping were performed using an FEI Tecnai G2 F20 S-Twin (acceleration voltage of $200 \mathrm{kV}$ ) electron microscope equipped with an X-ray energy-dispersive spectroscopy detector. The X-ray diffraction (XRD) patterns were collected using a Bruker D8 multiply crystals X-ray diffractometer $\left(6^{\circ} \mathrm{min}^{-1}\right)$. X-ray photoelectron spectroscopy (XPS) was performed on a PerkinElmer Physics PHI 5300 spectrometer. The surface areas and pore-size distributions were analyzed by $\mathrm{N}_{2}$ adsorption-desorption isotherms using a Make ASAP 2460 instrument. Raman spectroscopy was measured using a Renishaw inVia Raman spectrometer and 514-nm excitation wavelength.

\section{Electrochemical measurements}

All electrochemical measurements were conducted on a $\mathrm{CHI}$ $760 \mathrm{E}$ electrochemical workstation with a standard threeelectrode setup. The samples were tested on a rotating disk electrode (RDE; $0.196 \mathrm{~cm}^{2}$ in area) as the working electrode with $\mathrm{Ag} / \mathrm{AgCl}$ and $\mathrm{Pt}$ wire as the reference and counter electrodes, respectively. The electrochemical measurements for all samples (RuCoMn@NC, RuCo@NC, etc.) were performed under the same test conditions. Typically, $2 \mathrm{mg}$ of catalyst was dispersed in a mixture of $0.05 \mathrm{~mL}$ of Nafion ( $5 \mathrm{wt} \%), 0.20 \mathrm{~mL}$ of ethanol, and $0.25 \mathrm{~mL}$ of distilled water and sonicated for $30 \mathrm{~min}$ to form an ink solution. Then, $10 \mu \mathrm{L}$ of the ink was loaded onto the RDE surface (loading $\sim 0.2 \mathrm{mg} \mathrm{cm}^{-2}$ ) and dried at room temperature. The HER electrocatalytic activity of the samples was examined by obtaining the polarization curves using linear sweep voltammetry (LSV) with a scan rate of $5 \mathrm{mV} \mathrm{s}^{-1}$ at a rotational speed of $1600 \mathrm{rmin}^{-1}$ in $1.0 \mathrm{~mol} \mathrm{~L}^{-1} \mathrm{KOH}, 0.5 \mathrm{~mol} \mathrm{~L}^{-1} \mathrm{H}_{2} \mathrm{SO}_{4}$, and $1.0 \mathrm{~mol} \mathrm{~L}^{-1} \mathrm{PBS}$ solutions, respectively. Electrochemical impedance spectroscopy (EIS) was performed with alternating current $(\mathrm{AC})$ voltage of $5 \mathrm{mV}$ amplitude in the frequency range of $0.05-100,000 \mathrm{~Hz}$. Stability tests were performed by long-term chronoamperometric $i$ - $t$ curves under a constant voltage. The Tafel slope was obtained by linear fitting according to the Tafel equation $(\eta=a+b \log j$, where $\eta$ is the overpotential, $b$ is the Tafel slope, and $j$ is the current density). The electrochemical double-layer capacitance $\left(C_{\mathrm{dl}}\right)$ was determined by cyclic voltammetry $(\mathrm{CV})$, which was performed at a voltage range of 0.88 $0.98 \mathrm{~V}(v s$. reverse hydrogen electrode (RHE)) and scan rates of $10-60 \mathrm{mV} \mathrm{s}^{-1}$ in $1.0 \mathrm{~mol} \mathrm{~L}^{-1} \mathrm{KOH}$. The electrocatalytic activity of glucose oxidation of the samples was evaluated by CV (scan rate of $10 \mathrm{mV} \mathrm{s}^{-1}$ ) and amperometry $(i-t)$. The polarization curve potentials were obtained with $i R$ compensation and converted to RHE using Equation (1).

$$
\begin{aligned}
E_{\mathrm{RHE}}= & E_{\mathrm{Ag} / \mathrm{AgCl}}+0.059 \mathrm{pH} \\
& +E_{\mathrm{Ag} / \mathrm{AgCl}}^{0}\left(E^{0}{ }_{\mathrm{Ag} / \mathrm{AgCl}}=0.197 \mathrm{~V}\right) .
\end{aligned}
$$

For alkaline water/glucose electrolysis, the catalyst ink was prepared following the same procedure for preparing the working electrodes, and $10 \mu \mathrm{L}$ of the catalyst ink was uniformly coated on the surface of a glassy carbon electrode (GCE), which was used as both cathode and anode. $1.0 \mathrm{~mol} \mathrm{~L}^{-1} \mathrm{KOH}$ with $0.3 \mathrm{~mol} \mathrm{~L}^{-1}$ glucose solution was used as the anode chamber electrolyte and $1.0 \mathrm{~mol} \mathrm{~L}^{-1} \mathrm{KOH}$ solution was added to the cathodic compartment. The LSV curve was performed at a scan rate of $5 \mathrm{mV} \mathrm{s}^{-1}$. 


\section{RESULTS AND DISCUSSION}

Synthesis and characterizations of RuCoMn@NC

Fig. 1a illustrates the preparation process for $\mathrm{Ru}-\mathrm{Co}-\mathrm{Mn}$ alloy NPs on the N-doped carbon support. First, the rhombic dodecahedron-shaped ZIF-67 precursor was successfully synthesized (Fig. $1 \mathrm{~b}$ and Fig. S1). The ZIF-67 was pyrolyzed under $\mathrm{H}_{2} / \mathrm{Ar}$ atmosphere. The carbonized product was denoted as Co@NC, which retained the rhombic dodecahedron shape with densely distributed Co particles (Fig. 1c). The synthesized Co@NC was dispersed in $n$-hexane, and an aqueous solution containing $\mathrm{Ru}^{3+}$ and $\mathrm{Mn}^{2+}$ was dropped into the homogeneous dispersion and stirred to obtain Co@NC impregnated with $\mathrm{Ru}^{3+}$ and $\mathrm{Mn}^{2+}$ (denoted as Co@NC/ $\mathrm{Ru}^{3+}$ and $\mathrm{Mn}^{2+}$ ). The obtained sample was freeze-dried and thermally treated under $\mathrm{H}_{2} / \mathrm{Ar}$ atmosphere to obtain RuCoMn@NC. The content of ruthenium was 2.15 at $\%$, according to the XPS results (Table S1). In comparison, RuCo@NC was synthesized using the same method without the addition of $\mathrm{Mn}^{2+}$. The TEM images of RuCoMn@NC and RuCo@NC (Fig. 1d and Fig. S2) show similar size and shape as those of Co@NC, and there were metallic NPs homogeneously embedded on the carbon support. The HR-TEM image of RuCoMn@NC (Fig. 1e) shows clear lattice fringes with an interfringe distance of $0.235 \mathrm{~nm}$ located between the $\{211\}$ lattice spacing of MnCo $(0.257 \mathrm{~nm})$ and $\mathrm{RuCo}(0.083 \mathrm{~nm})$. The analysis revealed that the as-prepared catalyst was loaded with the $\mathrm{Ru}$ Co-Mn trimetallic alloy. High-angle annular dark-field scanning TEM (HAADF-STEM) and the corresponding elemental mapping images (Fig. 1f) show that $\mathrm{Ru}, \mathrm{Co}, \mathrm{Mn}, \mathrm{C}$, and $\mathrm{N}$ elements are homogeneously distributed, which indicates that the RuCoMn alloy NPs are uniformly embedded on the N-doped carbon polyhedra.

Fig. 2a shows the XRD spectra of Co@NC, RuCo@NC, and
RuCoMn@NC. A diffraction peak at $30^{\circ}$ was observed for the three samples, indicating the presence of carbon. Diffraction peaks at $51.8^{\circ}$ and $60.2^{\circ}$ for Co@NC are ascribed to the (111) and (200) planes of the cubic phase of metallic Co (JCPDS No. 15-0806), indicating the existence of Co metal. For the RuCo@NC, the diffraction peaks are close to the standard Ru diffraction peaks (JCPDS No. 06-0663), but they all shift toward higher angles, suggesting a change in lattice spacing due to the formation of RuCo alloy. The diffraction peaks of RuCoMn@NC are located between those of standard Ru, Co, and Mn (JCPDS No. 32-0637), indicating the successful fabrication of highly crystalline alloyed RuCoMn NPs, which is consistent with the HR-TEM results (Fig. 1e). The elemental composition and electronic states of the samples were further analyzed by XPS. The peaks at about 484.2 and $462.0 \mathrm{eV}$, corresponding to $\mathrm{Ru} 3 \mathrm{p}_{1 / 2}$ and $\mathrm{Ru} 3 \mathrm{p}_{3 / 2}$ (Fig. 2b), confirm $\mathrm{Ru}$ metal in both RuCo@NC and RuCoMn@NC [32]. The Co 2p spectrum of RuCoMn@NC (Fig. 2c) indicates that the presence of $\mathrm{Co}^{0}(778.7$ and $793.8 \mathrm{eV})[33,34]$. The peaks at 781.4 and $797.3 \mathrm{eV}$ with shakeup satellites (786.4 and $803.1 \mathrm{eV}$ ) correspond to $\mathrm{Co}-\mathrm{N}_{x}$ species [34-36]. RuCo@NC and Co@NC show the same type of deconvolution peak. However, compared with those of Co@NC, the peak positions of RuCo@NC and RuCoMn@NC shift positively, which indicates electron transfer from Co to $\mathrm{Ru}$ or $\mathrm{Mn}$ [37]. The Mn 2p spectrum (Fig. 2d) reveals the presence of metallic $(653.7$ and $641.3 \mathrm{eV})$ and ionic $(642.6 \mathrm{eV}) \mathrm{Mn}$, and the peak centered at $647.0 \mathrm{eV}$ is attributed to the shakeup satellite peak [38]. The pore structures for the RuCo@NC and RuCoMn@NC catalysts were characterized by $\mathrm{N}_{2}$ adsorptiondesorption measurements. The Brunauer-Emmett-Teller (BET) surface area of RuCoMn@NC is $110.35 \mathrm{~m}^{2} \mathrm{~g}^{-1}$ (Fig. 2e), which is higher than that of RuCo@NC $\left(87.86 \mathrm{~m}^{2} \mathrm{~g}^{-1}\right)$ (Fig. S3), implying that the formation of the trimetallic alloy with the introduction
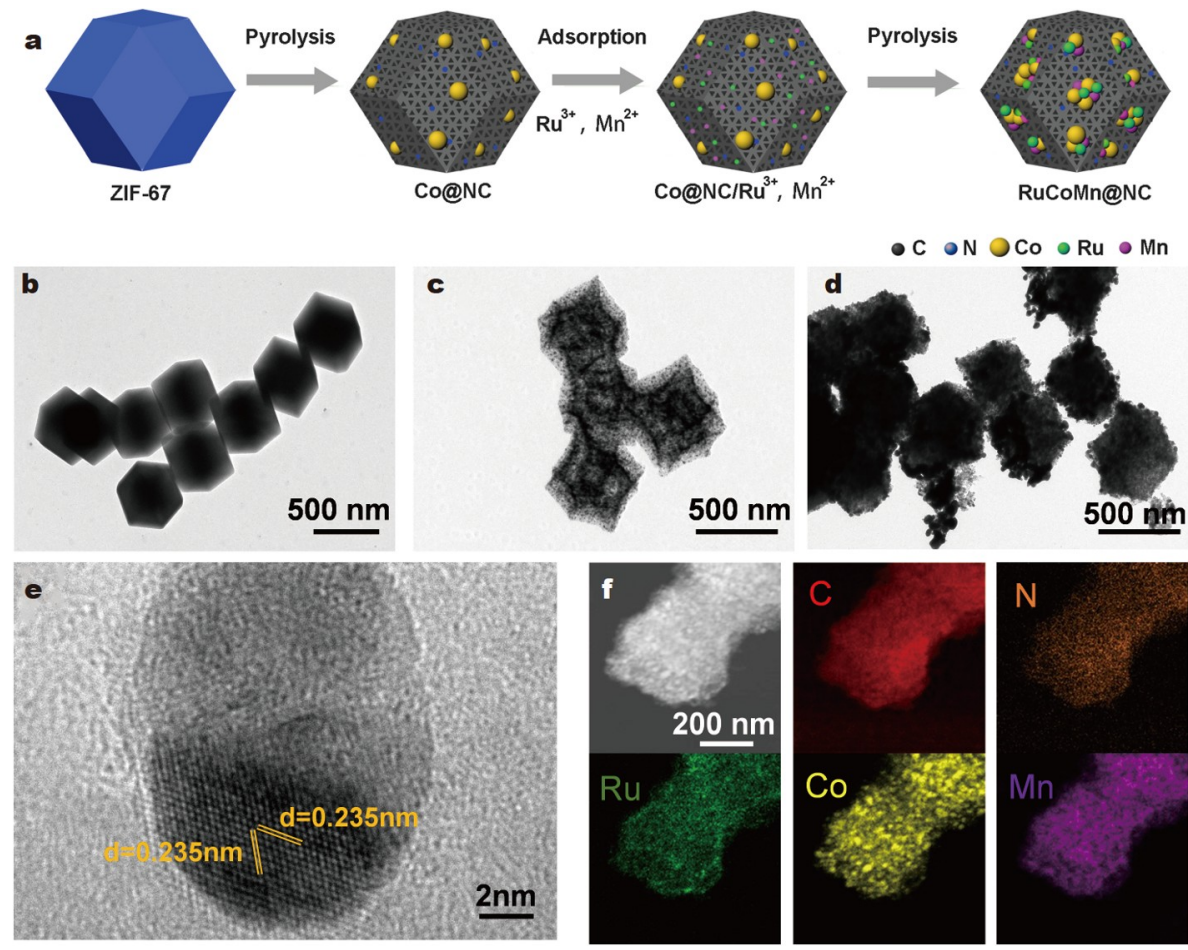

Figure 1 (a) Schematic illustration of the preparation process for RuCoMn@NC. TEM images of (b) ZIF-67, (c) Co@NC, and (d) RuCoMn@NC. (e) HRTEM image of RuCoMN@NC. (f) HAADF-STEM and the corresponding elemental mapping images of RuCoMn@NC. 

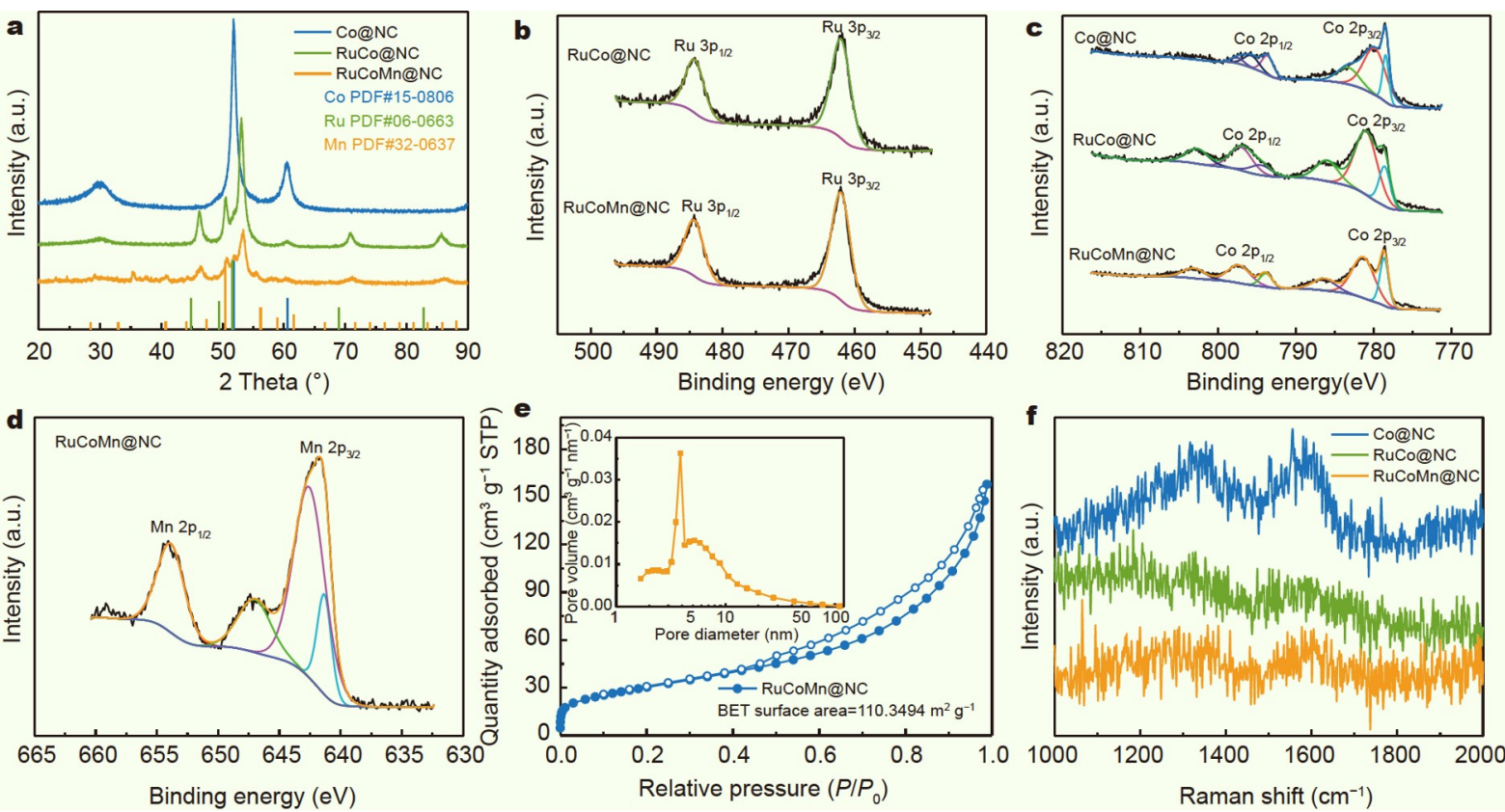

Figure 2 (a) XRD patterns of Co@NC, RuCo@NC, and RuCoMn@NC. XPS spectra of (b) Ru 3p, (c) Co 2p, and (d) Mn 2p of RuCoMn@NC. (e) N2 adsorption-desorption isotherm and the pore-size distribution of RuCoMn@NC. (f) Raman spectra of Co@NC, RuCo@NC, and RuCoMn@NC.

of $\mathrm{Mn}$ results in a higher surface area. Moreover, the pore-size distribution curves (inset of Fig. 2e) indicate the porosity of the RuCoMn@NC catalyst. The porosity and high surface area of the RuCoMn@NC catalyst enhance charge and mass transport during electrocatalysis [39]. The Raman spectrum shows two peaks at $\sim 1336$ and $1595 \mathrm{~cm}^{-1}$, representing the $\mathrm{D}$ band (defective carbon) and $\mathrm{G}$ band (graphitic carbon), respectively (Fig. 2f) [40].

\section{HER of RuCoMn@NC catalyst}

The HER catalytic performance of the as-synthesized RuCoMn@NC catalyst was first tested in a $1.0 \mathrm{ml} \mathrm{L}^{-1} \mathrm{KOH}$ aqueous solution. For comparison, several reference samples, including Co@NC, RuCo@NC, and the commercial 20\% Pt/C, were also examined. Fig. 3a shows the $i R$-corrected LSV curves of all samples at $1600 \mathrm{rmin}^{-1}$ with $1 \mathrm{mV} \mathrm{s}^{-1}$. RuCoMn@NC exhibits the highest HER activity with a low overpotential $(\eta)$ of only $38 \mathrm{mV}$ at a current density of $10 \mathrm{~mA} \mathrm{~cm}^{-2}$, which is lower than that of Co@NC $(215 \mathrm{mV})$, RuCo@NC $(67 \mathrm{mV})$, and even $\mathrm{Pt} / \mathrm{C}(64 \mathrm{mV})$. The Tafel slope was obtained to investigate the reaction kinetics during HER (Fig. 3b). The Tafel slope of RuCoMn@NC is approximately $34 \mathrm{mV} \mathrm{dec}^{-1}$, which is less than that of Co@NC (169 $\left.\mathrm{mV} \mathrm{dec}^{-1}\right), \mathrm{RuCo@NC}\left(53 \mathrm{mV} \mathrm{dec}^{-1}\right)$, and $\mathrm{Pt} / \mathrm{C}\left(35 \mathrm{mV} \mathrm{dec}^{-1}\right)$. This indicates that the Volmer-Tafel mechanism is the HER pathway [41-43]. The smaller Tafel slope of the RuCoMn@NC NPs indicates favorable HER kinetics. The EIS was employed to further investigate the kinetics between the electrode and electrolyte. RuCoMn@NC exhibits the smallest charge transfer resistance among all samples (Fig. 3c), indicating facilitated charge transfer. Therefore, it can promote the progress of HER. The durability of the RuCoMn@NC catalyst was evaluated by chronoamperometry. The catalyst retains $80.82 \%$ of the current density after a 10-h test (Fig. 3d). The TEM image of RuCoMn@NC after the durability test (Fig. S4) reveals that its size, morphology, and dispersion are maintained even after a long-term durability test. The $C_{\mathrm{dl}}$ of the catalysts was further measured by $\mathrm{CV}$ at a scan rate of $10-60 \mathrm{mV} \mathrm{s}^{-1}$ in a non-Faradic potential range (Fig. S5) to reflect the electrochemical surface area (ECSA) [44]. The $C_{\mathrm{dl}}$ of RuCoMn@NC $\left(15.3 \mathrm{mF} \mathrm{cm}^{-2}\right)$ is higher than that of Co@NC (3.3 $\left.\mathrm{mF} \mathrm{cm}^{-2}\right)$ and RuCo@NC $\left(2.8 \mathrm{mF} \mathrm{cm}^{-2}\right)$, indicating that the RuCoMn@NC catalyst has a larger electrochemical active surface area and more exposed active sites. These results demonstrate that the RuCoMn@NC NPs have excellent HER electrocatalytic activity and good stability in alkaline media.

Ideal HER electrocatalysts perform well over a wide $\mathrm{pH}$ range. Therefore, we further tested the HER activity of RuCoMn@NC in $0.5 \mathrm{~mol} \mathrm{~L}^{-1} \mathrm{H}_{2} \mathrm{SO}_{4}$ and $1.0 \mathrm{~mol} \mathrm{~L}^{-1} \mathrm{PBS}$. In the $0.5 \mathrm{~mol} \mathrm{~L}^{-1}$ $\mathrm{H}_{2} \mathrm{SO}_{4}$ medium, RuCoMn@NC exhibits a low overpotential of $119 \mathrm{mV}$ at a current density of $10 \mathrm{~mA} \mathrm{~cm}^{-2}$, which is slightly higher than that of $\mathrm{Pt} / \mathrm{C}(76 \mathrm{mV})$ but lower than that of RuCo@NC (150 mV) and Co@NC (273 mV) (Fig. 3e). The corresponding Tafel slope is $90 \mathrm{mV} \mathrm{dec}{ }^{-1}$, which is also higher than that of $\mathrm{Pt} / \mathrm{C}\left(27 \mathrm{mV} \mathrm{dec}^{-1}\right)$ but lower than that of RuCo@NC $\left(104 \mathrm{mV} \mathrm{dec}^{-1}\right)$ and Co@NC (122 $\left.\mathrm{mV} \mathrm{dec}^{-1}\right) \quad$ (Fig. 3f). RuCoMn@NC also shows better HER performance in neutral media than in acidic media. As shown in Fig. $3 g$, h, RuCoMn@NC exhibits a low overpotential at $10 \mathrm{~mA} \mathrm{~cm}^{-2}$ $(90 \mathrm{mV})$ and Tafel slope $\left(79 \mathrm{mV} \mathrm{dec}^{-1}\right)$ in $1.0 \mathrm{~mol} \mathrm{~L}^{-1} \mathrm{PBS}$, which are close to those of $\mathrm{Pt} / \mathrm{C}$ ( $82 \mathrm{mV}$ and $63 \mathrm{mV} \mathrm{dec}^{-1}$, respectively) and surpass those of RuCo@NC (254 mV and $174 \mathrm{mV} \mathrm{dec}^{-1}$, respectively) and Co@NC $\left(326 \mathrm{mV}\right.$ and $190 \mathrm{mV} \mathrm{dec}^{-1}$, respectively). Overall, these results confirm that RuCoMn@NC is a potential candidate for $\mathrm{pH}$-universal HER, and it outperforms the reported Ru-based electrocatalysts in alkaline solution or comparable to that in acidic and neutral solutions (Table S2). The excellent HER performance of RuCoMn@NC is superior to that of the Ru-based electrocatalysts reported so far, considering the lower overpotential, especially in alkaline media, or less content of noble metals (Table S2). To explore the possibilities of other ternary alloy systems, three other RuCo-based trimetallic electrocatalysts, including RuCoFe@NC, RuCoNi@NC, and 

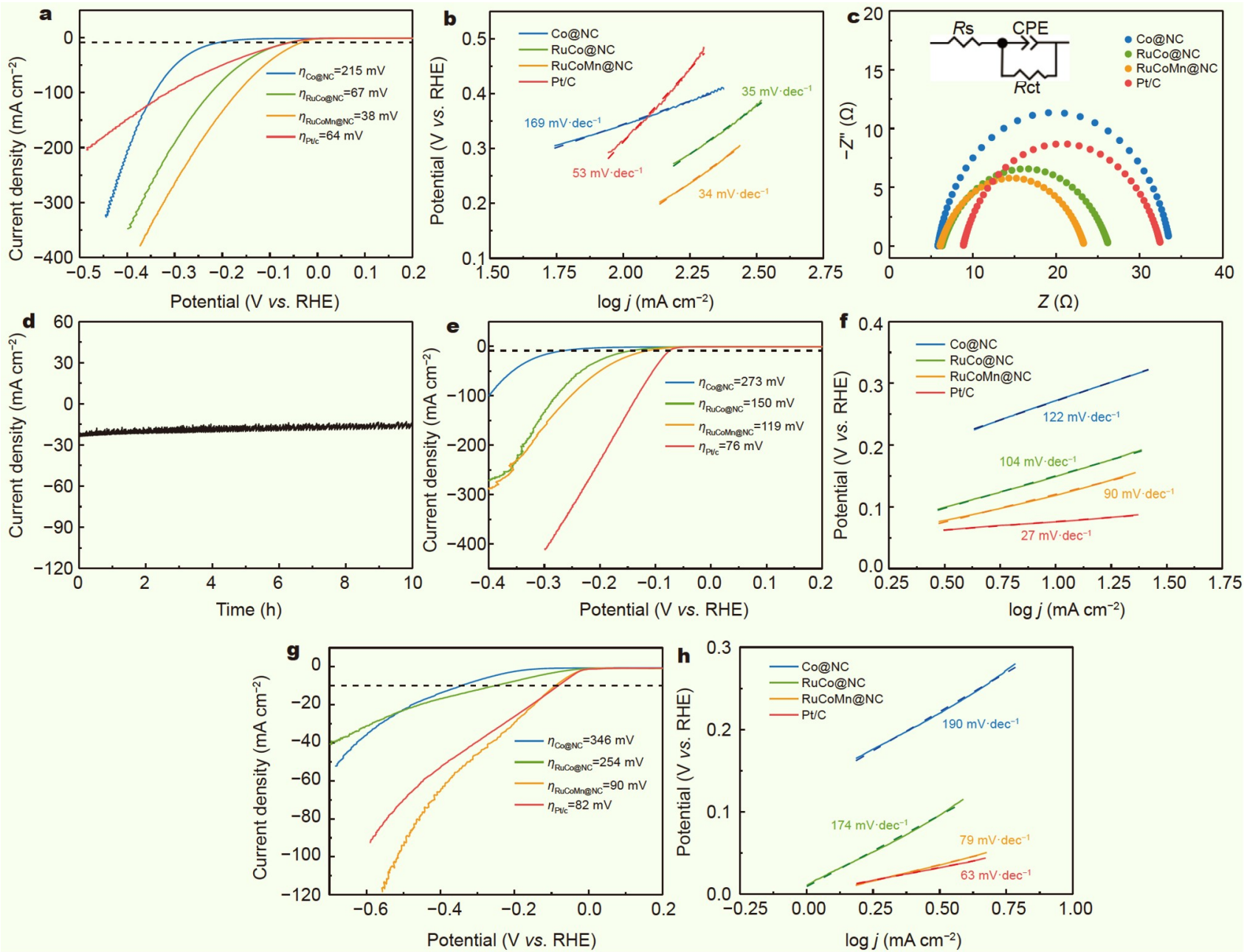

Figure 3 (a, e, g) iR-corrected LSV and (b, f, h) Tafel curves of RuCoMn@NC and the referenced samples in $1.0 \mathrm{~mol} \mathrm{~L}^{-1} \mathrm{KOH} 0.5 \mathrm{~mol} \mathrm{~L}^{-1} \mathrm{H}_{2} \mathrm{SO}_{4}$, and $1.0 \mathrm{~mol} \mathrm{~L}^{-1}$ PBS for HER at a scan rate of $1 \mathrm{mV} \mathrm{s}^{-1}$ and room temperature, respectively. (c) Nyquist plots of RuCoMn@NC and the referenced samples in $1.0 \mathrm{~mol} \mathrm{~L}^{-1} \mathrm{KOH}$. (d) Time-dependent current density curve over the RuCoMn@NC catalyst during electrolysis at $-0.2 \mathrm{~V}$ in $1.0 \mathrm{~mol} \mathrm{~L} \mathrm{KOH}^{-1}$.

RuCoCu@NC, were also synthesized via the same synthesis method. The samples show a similar morphology as that of RuCoMn@NC (Fig. S6). We evaluated the HER catalytic performance of the samples, and the results are shown in Fig. S7. In acidic and alkaline media, the performance of RuCoMn@NC is much higher than that of other ternary alloys, indicating that $\mathrm{Mn}$ is a better choice for participating in the formation of $\mathrm{RuCo}-$ based ternary alloy.

\section{GOR of RuCoMn@NC catalyst}

To further explore the potential of RuCoMn@NC as a redox bifunctional electrocatalyst, we evaluated the GOR activity in $1.0 \mathrm{~mol} \mathrm{~L}^{-1} \mathrm{KOH}$ electrolyte using the $\mathrm{CV}$ technique within the scope change of potential from 0.623 to $1.423 \mathrm{~V}$ ( $v s$. RHE) at a scan rate of $10 \mathrm{mV} \mathrm{s}^{-1}$. Fig. 4a shows the $\mathrm{CV}$ curves of RuCoMn@NC, RuCo@NC, and Co@NC in the absence (black line) and presence (red line) of glucose. In the absence of glucose, the CV curve of RuCoMn@NC in the electrolyte $\left(1.0 \mathrm{~mol} \mathrm{~L}^{-1} \mathrm{KOH}\right)$ shows a pair of well-defined redox peaks with the anodic and cathodic peaks at around 1.20 and $1.03 \mathrm{~V}$ ( vs. RHE), respectively, which can be attributed to the oxidation of tri-metal and reduction of the high valence of Co or Mn com- pound, respectively. After injecting glucose (concentration of glucose in alkaline solution is $3.0 \mathrm{mmol} \mathrm{L}^{-1}$ ), RuCoMn@NC shows a significantly higher current increase at the anodic peak than the RuCo@NC and Co@NC electrode, suggesting that RuCoMn@NC exhibits better catalytic activity toward GOR. Furthermore, oxidation current responses increase with the concentration of glucose in the RuCoMn@NC-modified GCE (Fig. 4b), demonstrating that glucose can be easily oxidized on the surface of the catalyst over a wide concentration window. The real-time amperometric detection of glucose on the RuCoMn@NC electrode was performed by successive stepwise addition of different amounts of glucose to a $\mathrm{KOH}$ solution at an applied potential of $1.223 \mathrm{~V}$ while being stirred (Fig. 4c). As expected, the RuCoMn@NC electrode shows a steep increase in current in response to the addition of glucose, indicating the sensitive and rapid response to GOR. The corresponding curve relating to the concentration and current response is plotted (Fig. 4d). Furthermore, the plot of the electrocatalytic current of glucose versus its concentrations in the intercepted range of $0.02-0.4 \mathrm{mmol} \mathrm{L}^{-1}$ is shown in the inset of Fig. $4 \mathrm{~d}$, and the corresponding linear equation is $I(\mu \mathrm{A})=7.0672 \mathrm{C}\left(\mathrm{mmol} \mathrm{L}^{-1}\right)+$ 4.3162, $R^{2}=0.9992$, with the calculated sensitivity of 
$36.06 \mu \mathrm{A} \mathrm{mmol}^{-1} \mathrm{~L} \mathrm{~cm}^{-2}$.

\section{Alkaline water/glucose electrolyzer}

From the electrochemical characterization of HER and GOR, the RuCoMn@NC catalyst is an efficient and robust bifunctional electrocatalyst for HER and GOR in alkaline solutions. As shown in Fig. 5a, a two-electrode electrolyzer was set up using RuCoMn@NC catalyst as both cathode and anode catalyst. LSV curves of the electrolyzer using $1.0 \mathrm{~mol} \mathrm{~L}^{-1} \mathrm{KOH}$, with or without $0.1 \mathrm{~mol} \mathrm{~L}^{-1}$ glucose, as electrolytes are compared in Fig. $5 \mathrm{~b}$. With glucose, the electrolyzer exhibits a higher electrolytic current density, requiring an applied voltage of $1.63 \mathrm{~V}$ at a current density of $10 \mathrm{~mA} \mathrm{~cm}^{-2}$, which is $270 \mathrm{mV}$ less than that of the electrolyzer without glucose. Therefore, replacing OER with GOR can reduce the potential of water electrolysis.

\section{CONCLUSIONS}

In summary, we synthesized Ru-Co-Mn trimetallic alloy NPs dispersed on $\mathrm{N}$-doped porous carbon polyhedra via the pyrolysis-adsorption-pyrolysis method using ZIF-67 as the precursor. Electrocatalytic tests show that the obtained RuCoMn@NC catalyst exhibits excellent bifunctional electrocatalytic properties for HER and GOR. The optimized RuCoMn@NC exhibits outstanding HER performance under all $\mathrm{pH}$ conditions, especially in alkaline media, with a low overpotential of $38 \mathrm{mV}$ at $10 \mathrm{~mA} \mathrm{~cm}^{-2}$ in $1.0 \mathrm{~mol} \mathrm{~L}^{-1} \mathrm{KOH}$, which is
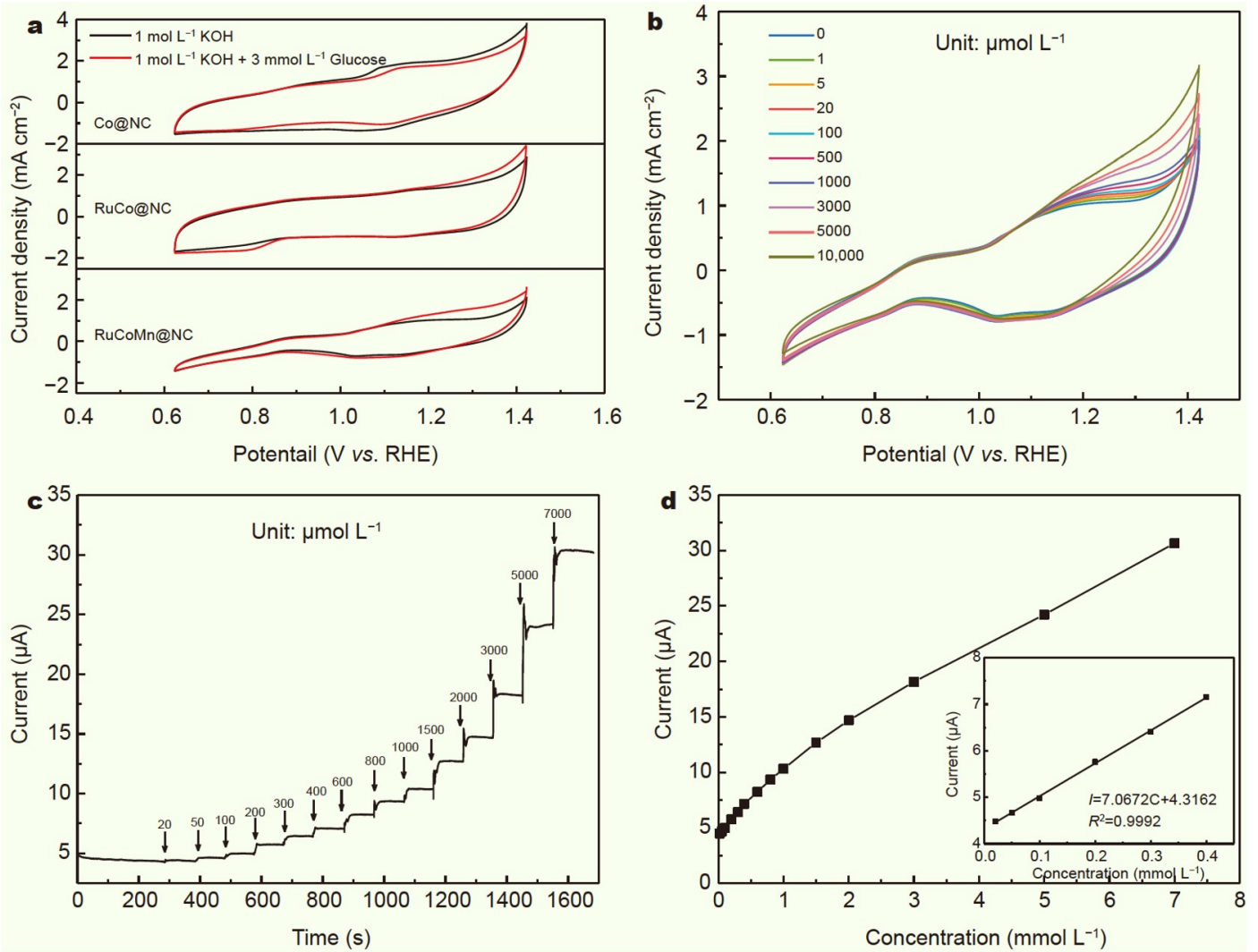

Figure 4 (a) CV curves of Co@NC, RuCo@NC, and RuCoMn@NC electrodes in the absence and presence of $3.0 \mathrm{mmol} \mathrm{L}^{-1}$ glucose in $1.0 \mathrm{~mol} \mathrm{~L}^{-1} \mathrm{KOH}$, respectively. (b) $\mathrm{CV}$ curves of the RuCoMn@NC electrode at different concentrations of glucose in $1.0 \mathrm{~mol} \mathrm{~L}^{-1} \mathrm{KOH}$. (c) Amperometric response of RuCoMn@NC at $1.223 \mathrm{~V}$ to successive addition of glucose in $1.0 \mathrm{~mol} \mathrm{~L}{ }^{-1} \mathrm{KOH}$. (d) Corresponding curve of current vs. glucose concentration with fitting curve and linear range (inset).
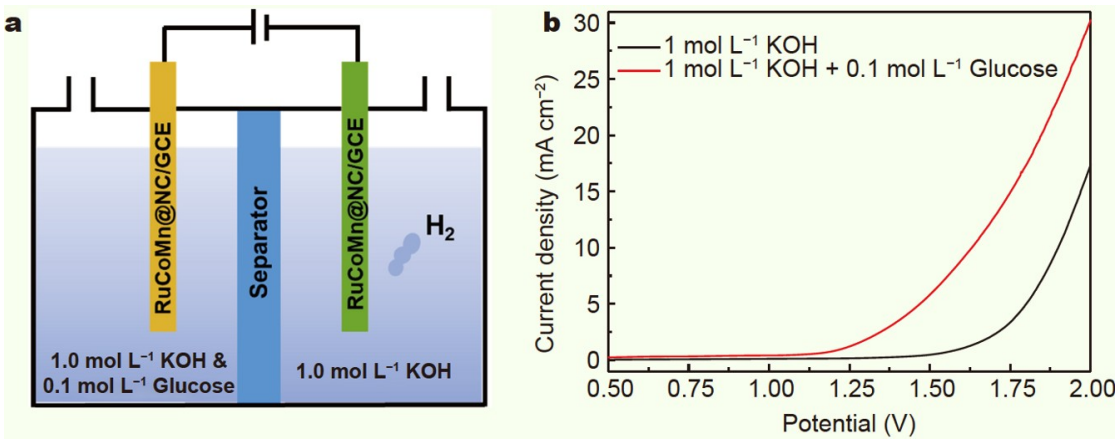

Figure 5 (a) Schematic diagram of alkaline water/glucose electrolyzer. (b) $\mathrm{LSV}$ plots of $\mathrm{RuCoMn} @ \mathrm{NC}$ in $1.0 \mathrm{~mol} \mathrm{~L} \mathrm{KOH}^{-1}\left(1.0 \mathrm{~mol} \mathrm{~L}^{-1} \mathrm{KOH}\right.$ and $0.1 \mathrm{~mol} \mathrm{~L}{ }^{-1}$ glucose) with a two-electrode system. 
comparable to that of $\mathrm{Pt} / \mathrm{C}$ and most state-of-the-art electrocatalysts. The excellent electrocatalytic activity of RuCoMn@NC is attributed to its unique hybrid structure and composition, with the combination of alloying effect, nanoscale particle size, large surface area, abundant mesopores, and the presence of conductive N-doped carbon support. Further, a two-electrode alkaline electrolyzer device pairing RuCoMn@NC as both the cathode and anode was assembled, and it shows a low cell voltage of $1.63 \mathrm{~V}$ to drive the current density of $10 \mathrm{~mA} \mathrm{~cm}^{-2}$ in a $1.0 \mathrm{~mol} \mathrm{~L}^{-1} \mathrm{KOH}+0.1 \mathrm{~mol} \mathrm{~L}^{-1}$ glucose electrolyte. This paper would contribute to the development of highly active and robust bifunctional electrocatalysts in water electrolysis using glucose or similar organic molecules.

Received 27 April 2021; accepted 4 June 2021; published online 9 August 2021

1 Wu F, Yang H, Bai Y, et al. Paving the path toward reliable cathode materials for aluminum-ion batteries. Adv Mater, 2019, 31: 1806510

2 Chen J, Pan A, Zhang W, et al. Melamine-assisted synthesis of ultrafine $\mathrm{Mo}_{2} \mathrm{C} / \mathrm{Mo}_{2} \mathrm{~N} @ \mathrm{~N}$-doped carbon nanofibers for enhanced alkaline hydrogen evolution reaction activity. Sci China Mater, 2021, 64: 11501158

3 Lin $\mathrm{C}$, Li H, Zhang $\mathrm{P}$, et al. Boosting water electrolysis with anodic glucose oxidation reaction over engineered cobalt nickel hydroxide nanosheet on carbon cloth. J Electroanal Chem, 2020, 861: 113946

4 Liu X, He J, Zhao S, et al. Self-powered $\mathrm{H}_{2}$ production with bifunctional hydrazine as sole consumable. Nat Commun, 2018, 9: 4365

5 Peng L, Zheng X, Li L, et al. Chimney effect of the interface in metal oxide/metal composite catalysts on the hydrogen evolution reaction. Appl Catal B-Environ, 2019, 245: 122-129

6 You B, Jiang N, Liu X, et al. Simultaneous $\mathrm{H}_{2}$ generation and biomass upgrading in water by an efficient noble-metal-free bifunctional electrocatalyst. Angew Chem Int Ed, 2016, 55: 9913-9917

7 Jiang $\mathrm{N}$, You B, Boonstra R, et al. Integrating electrocatalytic 5hydroxymethylfurfural oxidation and hydrogen production via Co-Pderived electrocatalysts. ACS Energy Lett, 2016, 1: 386-390

8 You B, Liu X, Jiang N, et al. A general strategy for decoupled hydrogen production from water splitting by integrating oxidative biomass valorization. J Am Chem Soc, 2016, 138: 13639-13646

9 You B, Liu X, Liu X, et al. Efficient $\mathrm{H}_{2}$ evolution coupled with oxidative refining of alcohols via a hierarchically porous nickel bifunctional electrocatalyst. ACS Catal, 2017, 7: 4564-4570

10 Du P, Zhang J, Liu Y, et al. Hydrogen generation from catalytic glucose oxidation by Fe-based electrocatalysts. Electrochem Commun, 2017, 83: $11-15$

11 Rabaey K, Rozendal RA. Microbial electrosynthesis-Revisiting the electrical route for microbial production. Nat Rev Microbiol, 2010, 8: 706-716

12 Zhang E, Xie $\mathrm{Y}, \mathrm{Ci} \mathrm{S}$, et al. Porous $\mathrm{Co}_{3} \mathrm{O}_{4}$ hollow nanododecahedra for nonenzymatic glucose biosensor and biofuel cell. Biosens Bioelectron, 2016, 81: 46-53

13 Gao K, Wang B, Tao L, et al. Efficient metal-free electrocatalysts from $\mathrm{N}$-doped carbon nanomaterials: Mono-doping and Co-doping. Adv Mater, 2019, 31: 1805121

14 Zhang E, Xie Y, Ci S, et al. Multifunctional high-activity and robust electrocatalyst derived from metal-organic frameworks. J Mater Chem A, 2016, 4: 17288-17298

15 Cheng Z, Fu Q, Han Q, et al. A type of $1 \mathrm{~nm}$ molybdenum carbide confined within carbon nanomesh as highly efficient bifunctional electrocatalyst. Adv Funct Mater, 2018, 28: 1705967

16 Zhang Y, Wang Y, Jia S, et al. A hybrid of NiMo- $\mathrm{Mo}_{2} \mathrm{C} / \mathrm{C}$ as non-noble metal electrocatalyst for hydrogen evolution reaction in an acidic solution. Electrochim Acta, 2016, 222: 747-754

17 Feng S, Li X, Huo J, et al. Controllable synthesis of $\mathrm{CoS}_{2} @ \mathrm{~N} / \mathrm{S}$-codoped porous carbon derived from ZIF-67 for as a highly efficient catalyst for the hydrogen evolution reaction. ChemCatChem, 2018, 10: 796-803

18 Vikraman D, Hussain S, Truong L, et al. Fabrication of $\mathrm{MoS}_{2} / \mathrm{WSe}_{2}$ heterostructures as electrocatalyst for enhanced hydrogen evolution reaction. Appl Surf Sci, 2019, 480: 611-620

19 Chu S, Chen W, Chen G, et al. Holey Ni-Cu phosphide nanosheets as a highly efficient and stable electrocatalyst for hydrogen evolution. Appl Catal B-Environ, 2019, 243: 537-545

20 Anandhababu G, Huang Y, Babu DD, et al. Oriented growth of ZIF-67 to derive $2 \mathrm{D}$ porous $\mathrm{CoPO}$ nanosheets for electrochemical-/photovoltage-driven overall water splitting. Adv Funct Mater, 2018, 28: 1706120

$21 \mathrm{Hu}$ G, Li J, Liu P, et al. Enhanced electrocatalytic activity of $\mathrm{WO}_{3} @$ NPRGO composite in a hydrogen evolution reaction. Appl Surf Sci, 2019, 463: 275-282

22 Gupta S, Patel N, Miotello A, et al. Cobalt-boride: An efficient and robust electrocatalyst for hydrogen evolution reaction. J Power Sources, 2015, 279: 620-625

23 Zhong H, Wang J, Zhang Y, et al. ZIF-8 derived graphene-based nitrogen-doped porous carbon sheets as highly efficient and durable oxygen reduction electrocatalysts. Angew Chem Int Ed, 2014, 53: $14235-14239$

24 Yan D, Dou S, Tao L, et al. Electropolymerized supermolecule derived $\mathrm{N}, \mathrm{P}$ co-doped carbon nanofiber networks as a highly efficient metalfree electrocatalyst for the hydrogen evolution reaction. J Mater Chem A, 2016, 4: 13726-13730

25 Ito $\mathrm{Y}$, Cong W, Fujita T, et al. High catalytic activity of nitrogen and sulfur co-doped nanoporous graphene in the hydrogen evolution reaction. Angew Chem Int Ed, 2015, 54: 2131-2136

26 Qu L, Zhang Z, Zhang $\mathrm{H}$, et al. Transformation from graphitic $\mathrm{C}_{3} \mathrm{~N}_{4}$ to nitrogen-boron-carbon ternary nanosheets as efficient metal-free bifunctional electrocatalyst for oxygen reduction reaction and hydrogen evolution reaction. Appl Surf Sci, 2018, 448: 618-627

27 Meng Z, Xiao F, Wei Z, et al. Direct synthesis of L10-FePt nanoparticles from single-source bimetallic complex and their electrocatalytic applications in oxygen reduction and hydrogen evolution reactions. Nano Res, 2019, 12: 2954-2959

28 Azhar A, Li Y, Cai Z, et al. Nanoarchitectonics: A new materials horizon for Prussian blue and its analogues. Bull Chem Soc Jpn, 2019, 92: 875-904

29 Wang HF, Chen L, Pang H, et al. MOF-derived electrocatalysts for oxygen reduction, oxygen evolution and hydrogen evolution reactions. Chem Soc Rev, 2020, 49: 1414-1448

30 Rao CNR, Pramoda K. Borocarbonitrides, $\mathrm{B}_{x} \mathrm{C}_{y} \mathrm{~N}_{z}, 2 \mathrm{D}$ nanocomposites with novel properties. Bull Chem Soc Jpn, 2019, 92: 441-468

31 Lv Y, Han M, Gong W, et al. Fe-Co alloyed nanoparticles catalyzing efficient hydrogenation of cinnamaldehyde to cinnamyl alcohol in water. Angew Chem Int Ed, 2020, 59: 23521-23526

32 Su J, Yang Y, Xia G, et al. Ruthenium-cobalt nanoalloys encapsulated in nitrogen-doped graphene as active electrocatalysts for producing hydrogen in alkaline media. Nat Commun, 2017, 8: 14969

33 Su CY, Cheng H, Li W, et al. Atomic modulation of FeCo-nitrogencarbon bifunctional oxygen electrodes for rechargeable and flexible allsolid-state zinc-air battery. Adv Energy Mater, 2017, 7: 1602420

34 Sultan S, Tiwari JN, Jang JH, et al. Highly efficient oxygen reduction reaction activity of graphitic tube encapsulating nitrided $\mathrm{Co}_{x} \mathrm{Fe}_{y}$ alloy. Adv Energy Mater, 2018, 8: 1801002

35 Liu S, Wang Z, Zhou S, et al. Metal-organic-framework-derived hybrid carbon nanocages as a bifunctional electrocatalyst for oxygen reduction and evolution. Adv Mater, 2017, 29: 1700874

36 Zhang W, Jiang X, Wang X, et al. Spontaneous weaving of graphitic carbon networks synthesized by pyrolysis of ZIF-67 crystals. Angew Chem Int Ed, 2017, 56: 8435-8440

37 Gao H, Zang J, Liu X, et al. Ruthenium and cobalt bimetal encapsulated in nitrogen-doped carbon material derived of ZIF-67 as enhanced hydrogen evolution electrocatalyst. Appl Surf Sci, 2019, 494: 101-110

38 Ji D, Sun J, Tian L, et al. Engineering of the heterointerface of porous carbon nanofiber-supported nickel and manganese oxide nanoparticle for highly efficient bifunctional oxygen catalysis. Adv Funct Mater, 


\section{0, 30: 1910568}

39 Liu Q, Tian J, Cui W, et al. Carbon nanotubes decorated with CoP nanocrystals: A highly active non-noble-metal nanohybrid electrocatalyst for hydrogen evolution. Angew Chem Int Ed, 2014, 53: 67106714

40 Chen Z, Wu R, Liu Y, et al. Ultrafine Co nanoparticles encapsulated in carbon-nanotubes-grafted graphene sheets as advanced electrocatalysts for the hydrogen evolution reaction. Adv Mater, 2018, 30: 1802011

$41 \mathrm{Pu} \mathrm{Z}$, Amiinu IS, Kou Z, et al. $\mathrm{RuP}_{2}$-based catalysts with platinum-like activity and higher durability for the hydrogen evolution reaction at all pH values. Angew Chem Int Ed, 2017, 56: 11559-11564

42 Mahmood J, Li F, Jung SM, et al. An efficient and $\mathrm{pH}$-universal ruthenium-based catalyst for the hydrogen evolution reaction. Nat Nanotech, 2017, 12: 441-446

43 Morales-Guio CG, Stern LA, Hu X. Nanostructured hydrotreating catalysts for electrochemical hydrogen evolution. Chem Soc Rev, 2014, 43: 6555-6569

44 Šljukić B, Vujković M, Amaral L, et al. Carbon-supported $\mathrm{Mo}_{2} \mathrm{C}$ electrocatalysts for hydrogen evolution reaction. J Mater Chem A, 2015, 3: 15505-15512

Acknowledgements This work was supported by the National Natural Science Foundation of China (52072035, 51631001, 21801015, 51902023 and 51872030), the Fundamental Research Funds for the Central Universities (2017CX01003), Beijing Institute of Technology Research Fund Program for Young Scholars, and the Joint R\&D Plan of Hong Kong, Macao, Taiwan, and Beijing (Z191100001619002). We acknowledge the support from Beijing Zhongkebaice Technology Service Co., Ltd. for TEM and XPS characterizations.

Author contributions Liu S performed the experiments and wrote the article. Wan X, Pan R, and Su M participated in the experiments. Zhang X and Li Y performed the data analysis. Zhang E, Liu J, and Zhang J proposed the experimental design. All the authors contributed to the general discussion. All the authors have given approval to the final version of the manuscript.

Conflict of interest The authors declare that they have no conflict of interest.

Supplementary information Supporting data are available in the online version of the paper.

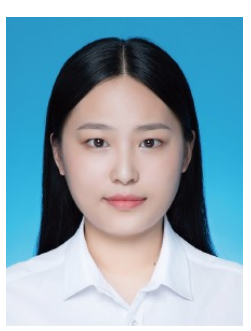

Shan Liu received her BE degree in 2019 from Beijing Forestry University and is currently an ME student at Beijing Institute of Technology (BIT). Her research interest focuses on composite nanomaterials and their applications in catalysis.

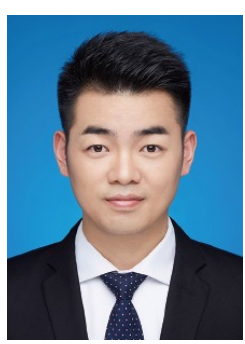

Erhuan Zhang received his PhD degree in 2020 from BIT and is currently a postdoctoral research fellow under the supervision of Prof. Yadong Li and Prof. Dingsheng Wang at Tsinghua University. His research interests cover advanced energy materials with a recent focus on single-atom catalysis.

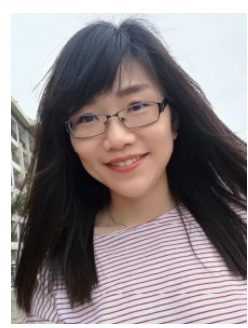

Jia Liu is currently an associate professor at the School of Materials Science and Engineering, BIT. She received her $\mathrm{PhD}$ degree from Dalian Institute of Chemical Physics, Chinese Academy of Sciences, in 2013. Before joining BIT in 2016, she worked as a postdoctoral research associate at the National Institute for Materials Science, Japan. Her research interests include the design and synthesis of hybrid nanocrystals for solar-to-fuel conversion systems.

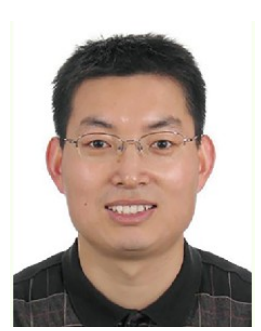

Jiatao Zhang received his $\mathrm{PhD}$ degree in 2006 from the Department of Chemistry, Tsinghua University. Currently, he is a Xu Teli Professor at the School of Materials and Engineering, BIT. He is also the director of Beijing Key Laboratory of ConstructionTailorable Advanced Functional Materials and Green Applications. His current research interest is in the inorganic chemistry of semiconductor-based hybrid nanostructures with novel optical and electronic properties for applications in solar-to-fuel conversion, catalysis, biology, and optoelectronics devices.

\section{双功能Ru-Co-Mn三元金属合金纳米催化剂用于氧化 还原电催化}

刘姗, 张二欢", 万晓冬, 潘容容, 李岳美, 张修铭, 苏梦瑶, 刘佳 ${ }^{*}$, 张加涛

摘要 电解水是最有前景的制氢方法之一, 但长期以来一直受到阳极 析氧反应(OER)迟缓的限制. 本文采用热解-吸附-热解工艺, 以ZIF-67 为前驱体，制备了 $\mathrm{Ru}-\mathrm{Co}-\mathrm{Mn}$ 三金属合金纳米颗粒 $(\mathrm{NPs})$ 负载在氮掺杂 碳载体上(RuCoMn@NC)的催化剂. 该催化剂在较宽的 $\mathrm{pH}$ 范围内对析 氢反应 (HER) 以及在碱性介质中对葡萄糖氧化反应 (GOR)都表现出良 好的电催化性能. 特别是RuCoMn@NC催化剂在碱性介质中表现出优 异的HER活性, 优于商用 $\mathrm{Pt} / \mathrm{C}$ 催化剂(20 wt \%), 并具有良好的电化学稳 定性. 进一步以RuCoMn@NC同时作为阴极和阳极组成双电极碱性电 解槽用于葡萄糖电解反应, 只需要 $1.63 \mathrm{~V}$ 的槽电压就可以达到 $10 \mathrm{~mA} \mathrm{~cm}^{-2}$ 的电流密度, 比全解水电解槽的所需电压低 $270 \mathrm{mV}$. 本工作 为合理开发高效的氧化还原电催化双功能催化剂提供了一种有效的方 法, 有利于节能电解制氢的发展. 